\title{
Etanercept-Induced Squamous Proliferations in a Patient With Porokeratosis
}

\author{
Maryam Liaqat, MD; Naomi Lawrence, MD; Steven Manders, MD
}

\section{PRACTICE POINTS}

- The use of biologics, particularly tumor necrosis factor $\alpha$ blockers, rarely are reported to induce skin cancer.

- Squamous cell carcinoma in the setting of biologic treatment would warrant a change of systemic medication.

\section{To the Editor:}

Etanercept is an immune-modulating drug used for the treatment of a variety of diseases including psoriasis, rheumatoid arthritis, and ankylosing spondylitis. It is an anti-tumor necrosis factor (TNF) fusion protein consisting of an extracellular domain of the p75 TNF receptor and the Fc portion of human IgG. ${ }^{1}$ Etanercept is well known for its immunosuppressive side effects. A handful of case reports have provided evidence of squamous cell cancers in the setting of etanercept therapy. The most comprehensive description was a case series by Brewer et $\mathrm{al}^{2}$ describing 4 patients with squamous cell carcinoma (SCC) that developed 1 to 17 months after the initiation of etanercept therapy. We present a case of a patient diagnosed with psoriasis and concomitant porokeratosis who developed multiple SCCs and squamous proliferations after initiation of etanercept therapy.

A 66-year-old man was referred to our clinic for treatment of psoriasis, as noted on a biopsy of the right ankle diagnosed several years prior. He was being treated with etanercept $50 \mathrm{mg}$ twice weekly. Other treatments included calcipotriene-betamethasone dipropionate, salicylic acid gel, intralesional triamcinolone, clobetasol, and urea $40 \%$. Physical examination revealed multiple erythematous tender nodules with hyperkeratotic scale distributed on the right arm and leg (Figure 1) that were concerning for SCC. Biopsies from 6 lesions revealed multiple SCC/keratoacanthomas (KAs) with verrucous features (Figure 2). Primers for human papillomavirus (HPV) 6, $11,16,18,31,33$, and 51 were all negative. At that time, etanercept was discontinued.

The patient was referred for Mohs micrographic surgery and underwent excision of several SCC lesions including an approximately $7-\mathrm{cm}$ SCC on the right ankle (Figure 1B). Positron emission tomography/computed tomography found hypermetabolic lymphadenopathy. A follow-up biopsy of the inguinal nodes identified no malignant cells. Given their multiplicity, the patient was initiated on a prolonged course of a retinoid with acitretin $35 \mathrm{mg}$ daily. The clearance of the large 7-cm lesion with a single stage of Mohs micrographic surgery directed suspicion to a pseudoepitheliomatous or HPV-induced cause for the lesions. Rereview of the original 6 biopsies indicated 1 definitive SCC on the right wrist, $2 \mathrm{KAs}$, and 3 that were most consistent with verruca vulgaris. At 1-year followup, most of the hyperkeratotic lesions had resolved with continued acitretin. Baseline porokeratosis lesions that were abundantly present on the arms and legs resolved by 1 -year follow-up (Figure 3A).

The link between classic porokeratosis and the development of squamous cell proliferations is well established. Ninomiya et $\mathrm{al}^{3}$ noted a possible mechanism of p53 overexpression in the epidermis of porokeratotic lesions that may make the lesions particularly susceptible

From the Department of Dermatology, Cooper University Hospital, Camden, New Jersey.

The authors report no conflict of interest.

Correspondence: Maryam Liaqat, MD, 3 Cooper Plaza, Ste 504, Camden, NJ 08103 (liaqat-maryam@cooperhealth.edu).

doi:10.12788/cutis.0301 

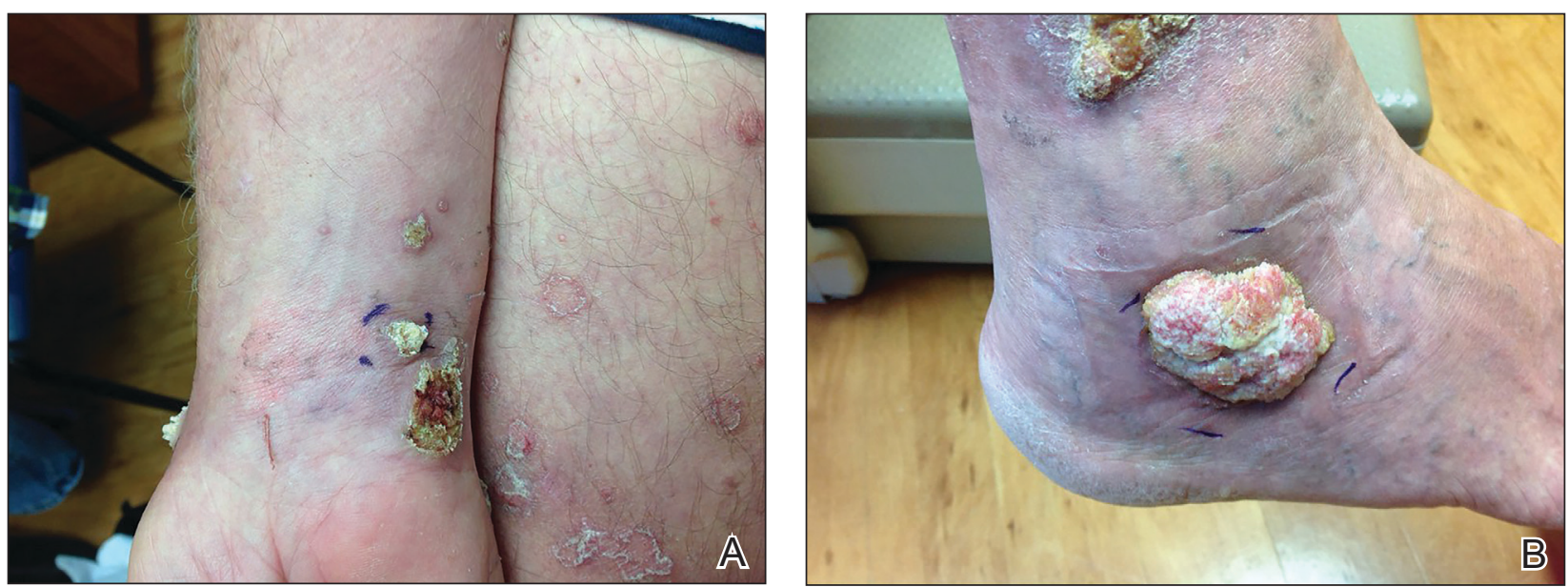

FIGURE 1. A, Erythematous tender nodules with hyperkeratotic scale on the wrist following use of etanercept. B, A 7-cm squamous cell carcinoma was present on the right ankle.
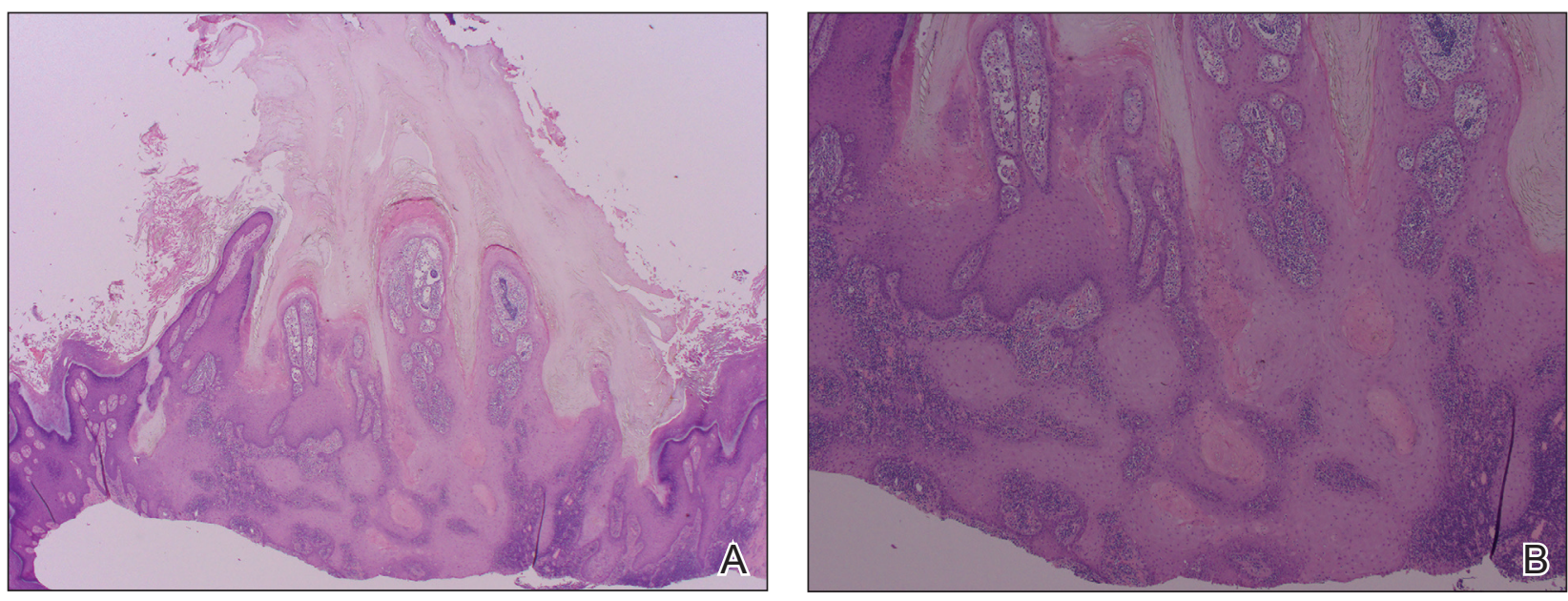

FIGURE 2. A and B, Histopathology of a lesion on the right medial wrist revealed atypical keratinocytes arranged in a digitate fashion, and some atypical cells were seen in the reticular dermis (H\&E, original magnifications $\times 10$ and $\times 10)$.

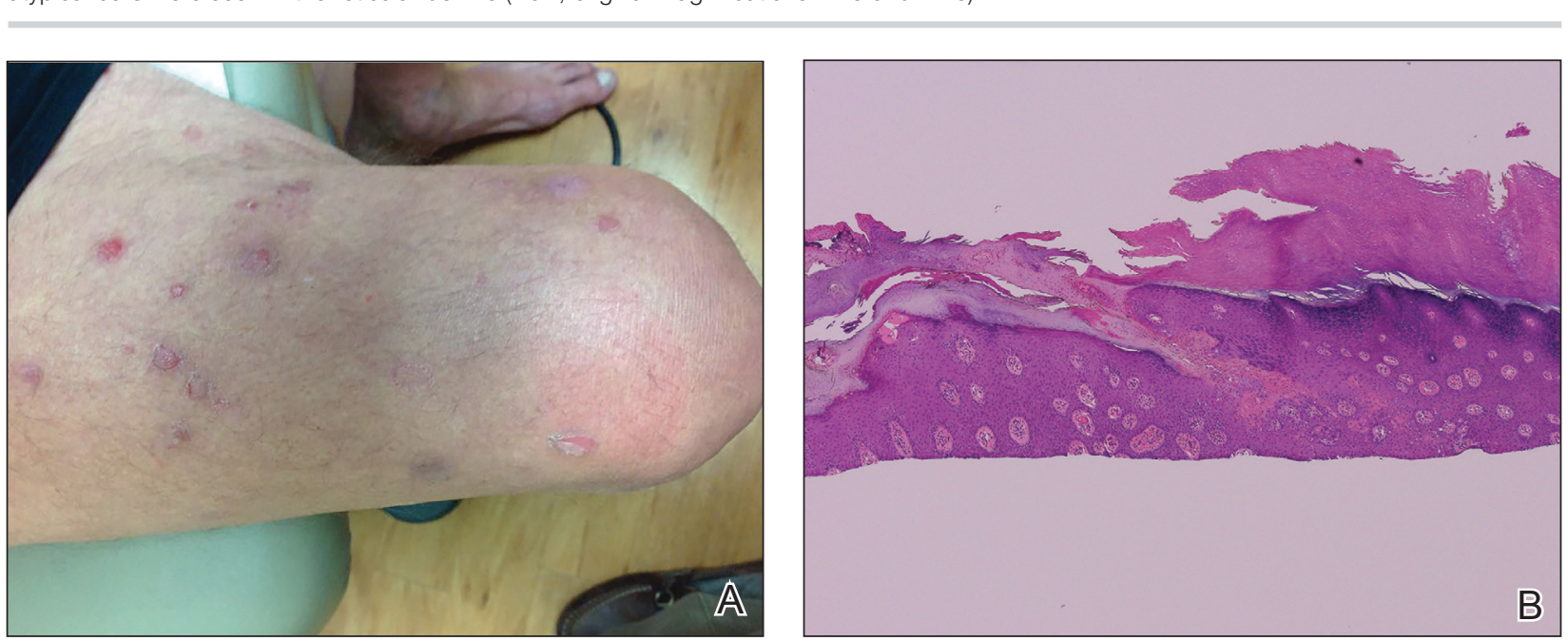

FIGURE 3. A, At 1-year follow-up after discontinuation of etanercept and initiation of acitretin, baseline porokeratosis lesions resolved.

$\mathrm{B}$, Histopathology of the right fourth finger revealed epidermal hyperplasia accompanied by columns of parakeratosis with underlying dyskeratosis $(H \& E$, original magnification $\times 10$ ). 
to the development of immunosuppression-induced SCC. Etanercept is an immune-modulating drug with wellknown immunosuppressive side effects including reactivation of HPV as well as the development of SCCs.

Our patient initially was diagnosed with psoriasis and etanercept was initiated. The presence of coexistent porokeratosis likely predisposed him to etanercept-induced squamous proliferations including 2 SCCs and verrucous lesions, with histologic features suggesting SCC/ KA. Histopathology revealed a cornoid lamella in SCC (Figure 3B), suggesting development of malignancy within epithelial clones, as noted by Lee et al. ${ }^{4}$

Targeted systemic therapies may lead to the formation of SCCs. The association between epidermal growth factor receptor (EGFR) kinase inhibitors and SCC formation is well known. For instance, sorafenib-a multikinase inhibitor that is downstream in the EGFR pathwayhas been noted to induce epidermal growths including KAs and SCCs. ${ }^{5}$ There has been no definitive causal relationship identified between the development of SCC and TNF- $\alpha$ inhibitors. It has been suggested that perhaps there is an unmasking effect, as subclinical SCC manifests after TNF- $\alpha$ inhibition that leads to SCC development.
Discontinuation of etanercept and resolution of lesions highlights a potential role of TNF- $\alpha$ inhibition and tumorigenesis of SCCs, especially in the background of porokeratosis. Vigilance for development of immunosuppression-induced malignancy, especially squamous cell proliferations, has become exceedingly important with exponentially increasing use of biologic therapies in medicine.

\section{REFERENCES}

1. Feldmann M, Charles P, Taylor P, et al. Biological insights from clinical trials with anti-TNF therapy. Springer Semin Immunopathol Springer Sem Immunopathol. 1998;20:211-228.

2. Brewer JD, Schott ARH, Roenigk RK. Multiple squamous cell carcinomas in the setting of psoriasis treated with etanercept: a report of four cases and review of the literature. Int J Dermatol. 2011; 50:1555-1559.

3. Ninomiya $Y$, Urano $Y$, Yoshimoto $K$, et al. p53 gene mutation analysis in porokeratosis and porokeratosis-associated squamous cell carcinoma. J Dermatol Sci. 1997;14:173-178.

4. Lee HR, Han TY, Son S-J, et al. Squamous cell carcinoma developing within lesions of disseminated superficial actinic porokeratosis. Ann Dermatol. 2011;23:536.

5. Kwon EJ, Kish LS, Jaworsky C. The histologic spectrum of epithelial neoplasms induced by sorafenib. J Am Acad Dermatol. 2009;61:522-527. 